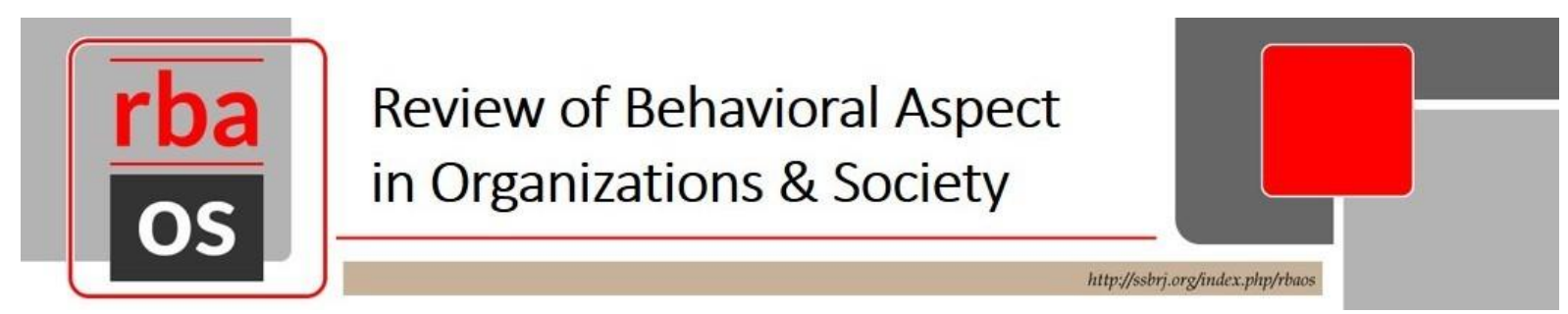

\title{
THE ROLE OF THE SPECIFICS OF SCHOOL INSTRUCTION IN RELATION TO MANIFESTATIONS OF SEMANTIC SENSORY ABILITIES AS MENTAL RESOURCES OF DIFFERENT TYPES OF MODALITY: TACTILE, TASTE, OLFACTORY AUDITORY AND VISUAL
}

\author{
Yana Sipovskaya Ivanovna, Russian Academy of Sciences, Russia
}

\begin{abstract}
The article examines the manifestations of semantic sensory abilities of different types of modality: tactile, auditory, taste, olfactory and visual, among older teens who study at two schools with different pedagogical orientation: a school with in-depth study of foreign languages and a school "Health", emphasizing attention to the physical and patriotic education of youth. The study involved 37 ninth-graders of the senior adolescence (15 years). Methodological basis of the study was "Visual semantics". The results of the study showed that there is no difference in the degree of manifestation of sensory abilities of tactile, auditory, taste, olfactory and visual types in adolescents. It probably depends on the degree of differentiation of semantic abilities to a greater extent than from the educational environment. These results emphasize both the theoretical and practical novelty of the proposed approach in view of a number of factors. First of all, a new method for diagnosing semantic sensory abilities had been approved. Furthermore, - studying regression (Alexandrov et al., 2017) of semantic sensory abilities as a variant of mental development and adaptive application of human mental resources. Finally, - data on the absence of differences in the degree of expression of sensory semantic abilities of all types in older adolescents. The established facts increase our understanding of the most basic, low-differentiated component of the conceptual experience - semantic abilities and one of its manifestations-sensory sensations, directing factors of heterogeneity in the semantic constructions of older adolescents to research. Moreover, the study of manifestations of semantic sensory sensitivity in other age periods is of particular relevance, which will allow us to reveal the temporal dynamics of the development of this component of conceptual experience.
\end{abstract}

Keywords: semantic sensory ability, modality, tactile, taste, olfactory, auditory, visual sensations, regression, degree of enrichment of the educational environment. 


\section{Introduction}

A number of factors, for example, the insufficient elaboration of the structure of conceptual experience and its components, emphasizes the urgency of the initiated research: conceptual, categorical and semantic abilities. Despite the fact that in M.A. Kholodnaya's research [2012: 116-127] in her conception of the structure of conceptual experience determines different manifestations of conceptual abilities; they do not receive further elaboration as metastructures in relation to their components:

- semantic abilities as psychic properties related to the productivity of semantic processes and revealing themselves in the processes of operating with the content of verbal signs within the framework of an individual mental lexicon and in the speed of operative processing of semantic information;

- categorical abilities as psychic properties related to the productivity of categorization processes and ensuring the attribution of the corresponding object to a certain category on the basis of transformations in a system of categorical attributes of different degrees of generalization;

- conceptual abilities as psychic properties related to the productivity of conceptualization processes and ensuring the possibility of generating some new mental contents not represented in the available information (identifying implicit connections and regularities, constructing metaphors, interpreting, modeling, thought experiment, etc.).

In the presented classification, the hierarchical structure of the most basic types of conceptual experience-semantic abilities - is not well developed, and in particular, such important manifestations as sensory semantic abilities have not been studied. Thus, there is a need to introduce a new conceptual apparatus, to which the concept of the semantic sensory capacity refers. In addition, the sensory capabilities of various types of modality are distinguished, namely: visual, auditory, tactile, olfactory and taste sensations. Accordingly, there is reason to talk about the semantic sensory abilities of the visual, auditory, tactile, olfactory and taste types. Along with this, there is a growing need for empirical studies to establish the importance of taking into account a number of factors potentially related to the manifestations of these abilities, for example, gender, age, environment, which forms the "development potential" (Vygotsky, 1984; 1999).

The theoretical and practical relevance of the work also emphasizes the introduction of a new educational standard for basic general education, which focuses on the formation of "universal educational activities" (UEA) among schoolchildren, one of whose blocks (instrumental) is impossible without mastering conceptual (and semantic in particular) abilities.

Speaking about the choice of the age category of participants in the study, one should point out the fundamental criticality of this period of a person's life in a number of spheres of his activity, including intellectual (Vekker, 1976).

Thus, variables of the study: the manifestations of the semantic sensory capabilities of different modes of modality: tactile, auditory, taste, olfactory and visual, and the educational environment of the participants in the study.

The subject of the study is the ratio of the manifestation of the semantic sensory abilities of different modes of modality: tactile, auditory, taste, olfactory and visual sensitivity, and the degree of enrichment of the participants' educational environment. The object of the study is older adolescents (median is 15 years). 
The purpose of the study: the disclosure of the specifics of the correlation between the features of the educational environment and the manifestations of the semantic sensory abilities of different modes of modality: tactile, auditory, taste, olfactory and visual, in the older adolescent age.

Theoretical hypothesis of the study: the degree of enrichment of the educational environment, in which the study participants are located, is related to the indicators of the semantic sensory abilities of different modes of modality: tactile, auditory, taste, olfactory and visual.

Research hypothesis: there are significant differences between older adolescents in the level of expression of the indicator of the semantic sensory abilities of different modes of modality: tactile, auditory, taste, olfactory and visual, depending on the degree of enrichment of the educational environment.

The research objective is to determine the ratio of the degree of enrichment of the educational environment in which the study participants are located and the manifestations of the semantic sensory abilities of different modes of modality: tactile, auditory, taste, olfactory and visual.

\section{Methodology}

Study participants: 37 schoolchildren at the age of 15 .

Modified method "Visual semantics" by Artemyeva (1980).

As there is a mechanism that regularly "packs" the experience of human interaction with the world in some special structures? We could talk about "perceptual universals" (Artemieva, 1999: 14), as Artemyeva called them in her study (Artemyeva, 1980).

The participant of the study on a white A4 sheet at the top is presented with a graphic image. It was necessary to answer the question in relation to each image: "What properties do you feel are inherent in this object? What is it? "(A list of these properties is written down). In total, five images were taken from the Artemieva(1999) set (Figure 1).
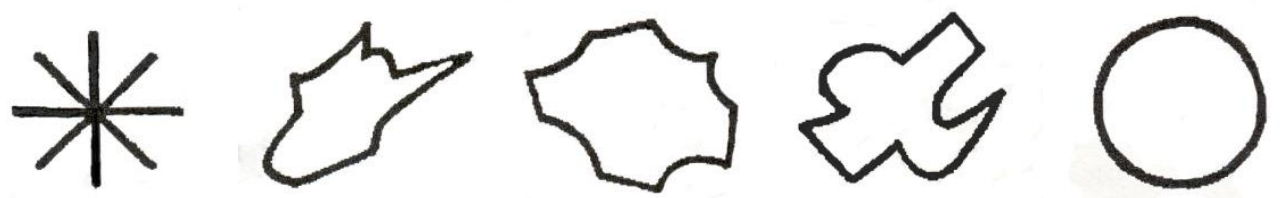

(Artemieva, 1999); compiled by the author.

Figure 1. Examples of graphic images in the "Visual semantics" technique

With the help of this technique, the ability to generate semantic features in the description of indefinite visual forms is evaluated.

Based on the analysis of the protocols, four types of semantic features were identified:

1) semantic signs of a sensory type (for example, cold, loud, prickly, etc.);

2) semantic signs of emotional-personal type (kind, cheerful, sad, affectionate, etc.);

3) semantic signs of a dynamic type (running, developing the speed of light, racing, jumping,etc.);

4) semantic signs of a logical type (dilapidated, complex, untidy, incomprehensible, tarnished, etc.). 
Following the tasks posed in this study, we considered exclusively the semantic features of the sensory type, subdividing them additionally according to the modalities into:

- semantic sensory signs of a tactile type (cold, soft, rough, etc.);

- semantic sensory attributes of taste type (bitter, sour, etc.);

- semantic sensory signs of olfactory type (pleasant odor, etc.);

- semantic sensory signs of auditory type (loud, noisy, sounding, etc.);

- semantic sensory attributes of the visual type (colorful, bright, etc.).

Indicators (calculated for each participant in the study): the number of sensory semantic features of each of the five types.

The study was conducted in 2 general schools in Moscow:

- School with in-depth study of English [Eng] (the number of participants in the study is 18 people);

- "School of Health", specializing in the physical and patriotic education of students [Health] (the number of participants in the study - 19 people).

Statistical processing was carried out using the methods of descriptive statistics and nonparametric methods of sample comparison, namely: Kruskel Wallace's method. The modern packages of statistical data processing (SPSS 20.1) were used.

\section{The results of the study and their discussion}

At the first stage of the statistical analysis, the study participants were divided into two groups, depending on the school in which they studied - a school with in-depth study of English, Eng; "School of Health", specializing in the physical and patriotic education of students, Health (Table 1).

Table 1. Ranked statistics: average ranks for boys and girls on the indicator of intellectual competence in various schools

\begin{tabular}{llll}
\hline Indicators & School & n & Average Rank \\
\hline \multirow{2}{*}{ Tactile } & Eng & 18 & 16,78 \\
& Health & 19 & 21,11 \\
Auditory & Eng & 18 & 20,08 \\
\multirow{2}{*}{ Taste } & Health & 19 & 17,97 \\
& Eng & 18 & 20,17 \\
Olfactory & Health & 19 & 17,89 \\
& Eng & 18 & 19,11 \\
& Health & 19 & 18,89
\end{tabular}

Note: "n" - is the number of participants in the study.

Further, the hypothesis was tested on the difference between older adolescents in secondary general education schools of various specializations, in terms of the manifestation of manifestations of semantic sensory abilities of different types of modality: tactile, taste, olfactory, auditory, and visual, Kraskel Wallace (Table 2). 
Table 2. Verification of the research hypothesis on the difference between adolescents studying in different schools in terms of the level of manifestation of the manifestation of semantic sensory abilities of different types of modality by the Kraskel-Wallace method

\begin{tabular}{|c|c|c|c|c|c|}
\hline \multirow[t]{2}{*}{ Indicators } & \multicolumn{5}{|c|}{ Variables } \\
\hline & Tactile & Visual & Auditory & Taste & Olfactory \\
\hline Chi-square & 1,505 & ,369 & ,789 & ,013 & 1,056 \\
\hline $\begin{array}{l}\text { Asymptotic } \\
\text { significance }\end{array}$ & ,220 & ,543 & ,374 & 910 & ,304 \\
\hline
\end{tabular}

Note: degree of freedom - 1 .

In accordance with the results, obtained in Table 2, conclusions were drawn regarding the hypotheses:

1) the hypothesis of the equality of samples of older adolescents in terms of the level of manifestation of the indicator of manifestations of semantic sensory abilities of the tactile type is plausible;

2) the hypothesis about the equality of samples of older adolescents in terms of the level of manifestation of the indicator of manifestations of the semantic sensory abilities of the visual type is plausible;

3 ) the hypothesis that the samples of older adolescents are equal in terms of the level of manifestation of the indicator of semantic sensory abilities of the auditory type is plausible;

4) the hypothesis about the equality of samples of older adolescents in terms of the level of expression of the manifestation of semantic sensory abilities of the olfactory type is plausible;

5) the hypothesis about the equality of samples of older adolescents in terms of the level of manifestation of manifestations of semantic sensory abilities of the taste type is plausible.

Thus, the obtained results point to the fact, that the presented study did not reveal differences in the level of sensory semantic abilities in older adolescents studying in schools with different pedagogical orientation. Accordingly, the specific nature of the educational environment has a statistically insignificant effect on the manifestation of such basic types of conceptual abilities as semantic sensory abilities. Probably, this result can be explained by the low differentiation of the construct of semantic abilities, which does not allow to reveal individual differences in the participants of the study. This level of conceptual abilities is the basis, the basis for more complex structures, highly differentiated manifestations of mental experience. In accordance with the concept of MA. Cold, semantic abilities lie at the heart of the hierarchical pyramid of conceptual experience, which, in turn, determines the operation of generalized and individual-personal concepts [7, 9, 10]. The latter, in turn, expand the intellectual capabilities of man (Vekker, 1976), causing high productivity of its activities (for example, intellectual competence).

In turn, we should point to the regression of the conceptual experience reflected in the results obtained in the form of sensory semantic structures (Sipovskaya, 2017). So, the higher cognitive abilities, for example conceptual or metacognitive abilities, differ in the degree of representation in the individual experience of study participants studying in schools with different pedagogical orientation. The same study did not reveal such a difference. Probably, the low degree of differentiation of semantic abilities, their emotionality plays a leading role in this aspect, determining the revealed regression. 
Thus, the results of the empirical study do not reveal the importance of taking into account the enrichment factor of the educational environment with respect to the sensory semantic abilities of the visual, tactile, olfactory, taste and auditory type in the older adolescence. This fact is probably due to equal or slightly different intellectual abilities that senior teenagers have from a specialized educational institution with in-depth study of foreign languages, compared to peers with a patriotic and sportive bias of pedagogical education in relation to low-grade semantic abilities.

\section{Conclusions}

The obtained results emphasize the importance of a careful analysis of the forms of expression of conceptual experience from highly organized conceptual abilities that classify categorical abilities to low-differentiated semantic abilities. The article does not reveal the fundamental importance of taking into account the specifics of the educational environment of older adolescents in determining the degree of formation of this kind of mental resources, such as sensory semantic abilities of various types of modality - visual, tactile, taste, auditory and olfactory. Thus, older adolescents in schools with different pedagogical orientation demonstrated no significant differences in the degree of manifestation of the manifestations of the semantic sensory abilities of all types of modality.

Thus, it can be concluded that the hypothesis set forth in this empirical study is false: significant differences between older adolescents studying at school with in-depth study of the English language are not revealed in the level of manifestations of the semantic sensory abilities of the visual, tactile, olfactory, auditory and taste type.

The obtained results deepen our understanding of the most basic, low-differentiated manifestations of the conceptual experience-semantic abilities, in particular, the semantic sensory abilities of sensations. The absence of the revealed differences between the manifestations of the semantic abilities of different types of modality - visual, tactile, olfactory, taste and auditory - indicates, on the one hand, the low-diffraction of the structures of semantic experience, and on the other hand, the fact that individual differences do not manifest themselves on such basic level of conceptual abilities. The last argument can be attributed to the support of the concept of M.A. Kholodnaya (2012) with respect to the hierarchical structure of conceptual experience. The established facts answer a number of questions and raise new ones, for example, the results indicate the need for a more careful analysis of the forms of the conceptual experience: highly organized conceptual abilities, differentiating and classifying categorical abilities and low-grade semantic abilities. Moreover, it is likely that other factors, such as the economic and social status of the study participants, also affect the findings. Do not underestimate the importance of expanding methodical and statistical methods and methods of data collection and processing.

\section{References}

Alexandrov, Y. I., Svarnik, O. E., Znamenskaya, I. I., Arutyunova, K. R., Kolbeneva, M. G., Krylov, A. K., \&Bulava, A. I. (2017). Regression as a stage of development. Moscow: Institute of Psychology of the Russian Academy of Sciences.

Artemieva, E. Y. (1999). Fundamentals of psychology of subjective semantics (I. B. Khanina, Ed.), Fundamental psychology series. Moscow, Russia: Science.

Artemieva, E. Y. (1980). Psychology of subjective semantics. Moscow, Russia: Publishing house of Moscow University. 
Sipovskaya, Y.I. (2015). Conceptual, metacognitive and intentional descriptors of intellectual competence in the senior adolescence.Bulletin of St. Petersburg State University,12(4), 22-31.

Sipovskaya, Y. I. (2017). Manifestations of regression in the structure of the conceptual experience of older adolescents. Journal of American Scientific, (14), 28-35.

Kholodnaya, M. A. (2002). The psychology of intelligence. St. Petersburg, Russia: Peter..

Kholodnaya, M. A. (2012). Psychology of conceptual thinking: from conceptual structures to conceptual abilities. Moscow, Russia: Institute of Psychology RAS.

Vekker, L. M. (1976). Psychological processes.Saint Petersburg, Russia: Publishing House of Leningrad State University..

Vygotsky, L. S. (1986). Scientific legacy in 6 volumes (M. G. Yarosheysky, Ed.).Moscow, Russia: Pedagogika. Vol. 6, 33-34.

Vygotsky, L. S. (1999). Pedagogical psychology. Moscow, Russia: Pedagogika-Press. 
Review of Behavioral Aspect in Organizations \& Society 16 